\title{
Concerted evolution of duplicate fla genes in Campylobacter
}

\author{
Richard J. Meinersmann and Kelli L. Hiett \\ Author for correspondence: Richard Meinersmann. Tel: +1 706546 3236. Fax: +1 7065463633. \\ e-mail:rmeiners@asrr.arsusda.gov
}

USDA Agricultural Research Service, Russell Research Center, PO Box 5677, Athens, GA 30604-5677, USA

\begin{abstract}
Campylobacters have two similar copies ( $\mathrm{flaA}$ and flaB) of their flagellin gene. It has been hypothesized that the two copies can serve for antigenic phase variation. Analysis of polymorphisms within aligned multiple DNA sequences of the Campylobacter flagellin genes revealed high pairwise homoplasy indexes between flaB/flaB pairs that were not observed between any flaA/flaA pairings or flaAlflaB pairings. Thus it seems there are constraints on the sequence of flaB that distinguish it from flaA. Nevertheless, segments of the two genes that are highly variable between strains are conserved between the flaA and flaB copies of the genes within a strain. The patterns of synonymous and non-synonymous differences suggest that one segment of the flagellin sequence is under selective pressure at the amino acid sequence level. Another segment of the protein is maintained within a strain by conversion or recombination. Comparisons of strict consensus amino acid sequences did not reveal any motifs that are uniquely FlaA or FlaB, but there are differences between FlaA and FlaB in those amino acids available for post-translational modification. The observed pattern of concerted evolution of portions of a structural gene is an unusual finding in bacteria and should be searched for with other duplicated genes. Concerted evolution was unexpected for genes involved in phase variation since it minimizes the antigenic repertoire that can be expressed by a single clone in the face of the host immune response.
\end{abstract}

Keywords: Campylobacter, flagellin, concerted evolution, DNA multiple-sequence analyses

\section{INTRODUCTION}

Gene duplication followed by specialization of each copy is thought to be a major mechanism of the evolution of genes (Jensen \& Gu, 1996). Gene duplication initially results in the production of paralogues with a high degree of similarity. In time, each gene accumulates differences or mutations independently of the other copy. Consequently, the genes diverge in their sequences. Duplicate genes with a high degree of similarity presumably either arose recently, or are subjected to selective pressures which maintain the similarity. Concerted evolution is observed when paralogous members of a gene family within a species diverge at a rate slower than the homologous genes in other species. Concerted evolution has been detected in several eukaryotic multicopy gene families (Li, 1997). This indicates that there are mechanisms to homogenize within a species the sequence of genes that are undergoing concerted evolution. Examples of concerted evolution in bacteria include complete operons such as the
rRNA operons (Mattatall et al., 1996), entire genes such as tufA and tufB in Salmonella typhimurium (Abdulkarim \& Hughes, 1996) or catlJF and pcalJF in Acinetobacter calcoaceticus (Kowalchuk et al., 1995), and tandem repeats within genes, such as those seen in alpha C protein of group B streptococci (Madoff et al., 1996).

The genes encoding flagellin in Campylobacter (flaA and $f l a B$ ) serve as an opportunity to further study gene duplication. The sequences for both $f l a A$ and $f l a B$ have been published for two isolates of Campylobacter jejuni (Khawaja et al., 1992; Nuijten et al., 1990) and one isolate of Campylobacter coli (Logan et al., 1989). The coding regions for the fla $A$ and $f l a B$ sequences were $1722-1731$ bases in length and the $f l a B$ sequence is separated from fla $A$ by approximately 180 bases. In each pair of genes from a single isolate, flaA differed from the flaB by about $5 \%$. Variation of each of the two genes from one strain to another was as much as $30 \%$, with most of the variation concentrated in a central region 
about a third the length of the gene (Khawaja et al., 1992; Logan et al., 1989; Nuijten et al., 1990). We have recently completed DNA sequence analyses of flaA from 15 Campylobacter isolates and verified the presence of a major hypervariable region from approximately base 700 to base 1450 , and a short variable region between bases 450 to 600 (Meinersmann et al., 1997). In Salmonella, nanostructure analyses of the flagellin demonstrated that similar variable regions are located within domains of the protein that are surface-exposed (Yamashita et al., 1995). We expect that the flagellins from Campylobacter have a similar conformational structure. It has been hypothesized that the second copy of fla serves as a potential donor for reassortment and recombination of the DNA as a mechanism for creating new antigenic variants for immune avoidance (Alm et al., 1993). Wassenaar et al. (1993) supported this hypothesis by selecting a variant in which $f l a B$ apparently replaced a defective flaA.

A $\sigma^{28}$-like promoter site has been identified for $f l a A$ and a $\sigma^{54}$-like site has been identified for flaB (Guerry et al., 1990; Nuijten et al., 1990). An inverted repeat suggestive of a transcription terminator was found downstream from the flaA and upstream of the $f l a B$ promoter (Khawaja et al., 1992; Logan et al., 1989; Nuijten et al., 1990). Different environmental factors appear to affect the separate promoters and, in culture-grown cells, $f l a B$ is expressed at a rate lower than that of flaA (Guerry et al., 1990) or not at all (Nuijten et al., 1990). Insertional mutagenesis of flaA yields cells with a truncated flagellum, greatly reduced motility, and reduced ability to colonize animals (Nachamkin et al., 1993; Wassenaar et al., 1991, 1993). Insertional mutagenesis of $f l a B$ yields cells that have flagella with apparently normal morphology and colonization ability and, perhaps, slightly decreased motility.

The high similarity between $f l a A$ and $f l a B$ in individual strains suggests that duplication of the gene is a relatively recent event. However, given the strain-to-strain variation of the fla genes, this conclusion is not clear. This study was undertaken to determine if patterns of evolution could be determined to explain the maintenance of a pair of highly similar fla genes within each strain of Campylobacter. We expected that the hypothesis that the duplicates provided variants for phase variation was correct and we wanted to determine the extent of variation that mechanism would create. However, the results indicated that the evolution of the fla $A$ and flaB genes in Campylobacter is coordinated. The residues that are predicted to be exposed are conserved between the flaA and $f l a B$ of a particular isolate while the same region has a high variability between strains. At the same time, there are specific portions of the sequence that distinguish fla $A$ from $f l a B$ and are conserved between strains.

\section{METHODS}

DNA sequences. DNA sequences for $f l a A$ and $f l a B$ for strains VC167, TGH9011 and 81116, and the flaA sequence for strain IN1, were obtained from the GenBank database (accession nos M26945, Z29327, J05635 and X57173, respectively). Sequences for $f l a A$ and flaB of strain NCTC 11186 were obtained by BLAST search of the genomic database at the Sanger Centre (http://www.sanger.ac.uk/Projects/ $\mathrm{C}$ jejuni/). The sequence generation for flaA of the remaining strains has been described previously (Meinersmann et al., 1997). The GenBank accession numbers are: strain A74/O, AF050184; strain D772, AF050185; strain D935, AF050186 (flaA) and AF050187 (flaB); strain D2290, AF050188 (flaA) and AF050189 (flaB); strain D2640, AF050190; strain D2677, AF050191; strain L17, AF050192 (flaA) and AF050193 (flaB); strain L19, AF050194; strain SSU9894, AF050195; strain SSU9896, AF050196; strain D1118, AF050197.

To obtain the DNA sequences for $f l a B$, sequences upstream of $f l a B$ were first determined as described below in order to produce additional information on the non-coding intergenic region. Template DNA was generated by PCR using primers that annealed to a conserved portion of the $3^{\prime}$ end of fla $\mathrm{A}$ paired with a downstream primer that annealed to a conserved portion at the $5^{\prime}$ end of the known flaB sequences. The products of these PCR reactions were ligated into a T/A cloning vector and sequenced as described by Meinersmann et al. (1997). An upstream primer was then designed based on a conserved portion of the intergenic sequence upstream of the $f l a B$ start codon. This was paired with a primer that was designed to complement conserved sequence $3^{\prime}$ distal to the flaB coding region of aligned sequences for strains VC167, TGH9011 and 81116. These primers were used in a PCR to amplify the entire $f a B$ and a small amount of flanking sequence at both ends under permissive conditions (i.e. with an annealing temperature $5^{\circ} \mathrm{C}$ lower than expected optimum), and the products were sequenced as described by Meinersmann et al. (1997) utilizing the same primers as were used for flaA sequencing. The sequences of this final PCR product were used for the flaB data in the analyses described below.

Computational analyses. Alignments were made of the DNA sequence of flaA from 16 strains or flaB from seven strains using CLUSTALX (Thompson et al., 1994) and the alignments were manually edited to move gaps inserted by the alignment program so that they did not interrupt the codon reading frame. Alignment scores were either unchanged or improved by such editing. The aligned sequences were analysed with PAup version 4.0b2a (Swofford, 1999) to reconstruct possible phylogenetic relationships and produce homoplasy indexes [homoplasy index $=1$ minus (the minimum possible number of steps between two individuals on a tree divided by the number of steps observed in the reconstructed tree between those two individuals); see Discussion for further details on homoplasy]. The aligned sequences were subjected to Sawyer's runs test (Sawyer, 1989), which looks for substitution patterns that are statistically consistent with recombination events, implemented in the program GENECONV version 1.02 (distributed by S. Sawyer at http://www.math.wustl.edu/ $\sim$ sawyer). This program performs the Bonferroni correction of Karlin-Altschul $P$ values, which is the most conservative method of determining the $P$ values.

Alignments were analysed in the MULTICOMP program (Reeves et al., 1994) using the algorithm developed by $\mathrm{Li}$ (1993) for determining synonymous and non-synonymous substitutions. $K_{\mathrm{s}}$ is the synonymous substitution rate and $K_{\mathrm{A}}$ is the nonsynonymous substitution rate expressed as the corrected number of substitutions per site. Rates greater than 1.00 are not real, but are due to corrections for possible multiple substitutions at a single site and remain proportional to the rate of neighbouring substitutions. The analyses were performed with a window size of 30 bases (10 codons). Use of 
Table 1. Pairwise distance and homoplasy matrix for Campylobacter flaA and flaB DNA sequences

Data above-right of the diagonal are the numbers of different nucleotide bases between paired sequences. Data below-left of the diagonal are the homoplasy indexes for each pair. Data in the upper-right quadrant and lower-left quadrant are flaA-flaB comparisons. Upper left data are $f l a B-f l a B$ and lower right are flaA-flaA comparisons. Boxed data are within-strain flaA-flaB comparisons. Data for strains with only flaA data are omitted from the table.

\begin{tabular}{|c|c|c|c|c|c|c|c|c|c|c|c|c|c|c|}
\hline & 1 & 2 & 3 & 4 & 5 & 6 & 7 & 8 & 9 & 10 & 11 & 12 & 13 & 14 \\
\hline 1. D2290 flaB & & 2 & 2 & 78 & 81 & 248 & 271 & 97 & 100 & 87 & 147 & 151 & 293 & 315 \\
\hline 2. $\mathrm{L} 17 \mathrm{flaB}$ & 0 & & 2 & 78 & 81 & 248 & 271 & 97 & 100 & 87 & 147 & 151 & 293 & 315 \\
\hline 3. $11168 \mathrm{flaB}$ & 0 & 0 & & 76 & 81 & 246 & 271 & 95 & 98 & 87 & 145 & 151 & 291 & 315 \\
\hline 4. TGH9011 flaB & 134 & 134 & 134 & & 103 & 242 & 259 & 145 & 144 & 139 & 85 & 163 & 289 & 307 \\
\hline 5. D935 flaB & 114 & 114 & 112 & 110 & & 259 & 268 & 150 & 153 & 150 & 174 & 78 & 300 & 314 \\
\hline 6. $81116 \mathrm{flaB}$ & 164 & 164 & 164 & 130 & 154 & & 177 & 297 & 296 & 297 & 297 & 301 & 89 & 235 \\
\hline 7. VC167 flaB & 162 & 162 & 160 & 134 & 166 & 86 & & 326 & 325 & 318 & 316 & 316 & 240 & 116 \\
\hline 8. D2290 flaA & 18 & 18 & 18 & 32 & 10 & 80 & 72 & & 3 & 10 & 76 & 82 & 248 & 276 \\
\hline 9. L17 flaA & 18 & 18 & 18 & 36 & 10 & 84 & 76 & 0 & & 13 & 75 & 81 & 247 & 275 \\
\hline 10. 11168 flaA & 30 & 30 & 28 & 40 & 12 & 82 & 82 & 0 & 0 & & 74 & 86 & 248 & 272 \\
\hline 11. TGH9011 flaA & 30 & 30 & 30 & 0 & 4 & 40 & 42 & 66 & 70 & 70 & & 104 & 248 & 266 \\
\hline 12. D935 flaA & 8 & 8 & 6 & 14 & 0 & 76 & 82 & 42 & 46 & 40 & 38 & & 252 & 266 \\
\hline 13. 81116 flaA & 116 & 116 & 116 & 80 & 110 & 4 & 20 & 126 & 130 & 128 & 86 & 122 & & 198 \\
\hline 14. VC167 flaA & 92 & 92 & 90 & 60 & 94 & 2 & 0 & 96 & 100 & 102 & 66 & 106 & 36 & \\
\hline
\end{tabular}

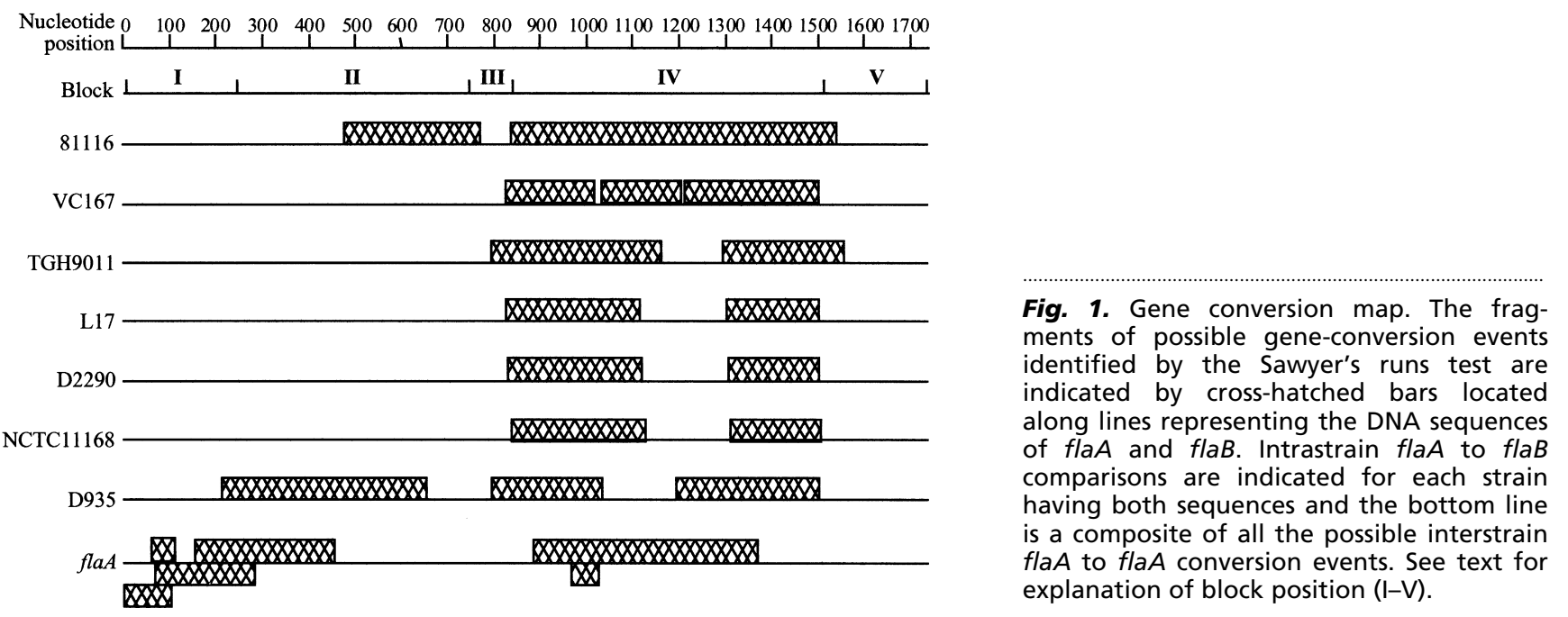

longer windows gave less saturated results but the resolution was less than desired. To analyse synonymous and nonsynonymous substitutions from flaA to $f l a B$ within strains, the sequences for seven strains were aligned pairwise for each strain, and each pair was analysed with the sITES program (distributed by Jody Hey, Rutgers University) to show the sites of synonymous and non-synonymous substitutions for each sequence pair. Aligned sequences were analysed with PAUP* 4.0b2a (Swofford, 1999) to construct the most parsimonious relationships and the homoplasy indexes were also determined with PAUP*.

Peptide sequences. The DNA sequences were translated to peptide sequences that were then aligned. One hundred per cent consensus peptide sequences were generated for the aligned $f l a A$ sequences and the aligned $f l a B$ sequences. Positions in which a consensus was not achieved were marked with an $\mathrm{X}$, unless all the residues were in the same pam250S group of similar amino acids (Dayhoff et al., 1978), in which case the position was marked 0 for Ala, Gly, Pro, Ser, Thr; 1 for Asp, Glu, Asn, Gln, Asx, Glx; 2 for His, Lys, Arg; 3 for Ile, Leu, Met, Val; 4 for Phe, Trp, Tyr; or 5 for Cys. The two consensus sequences were aligned and a new consensus was generated.

\section{RESULTS}

The DNA sequences of flaA for 16 isolates of Campylobacter (15 isolates of C. jejuni and one isolate of C. coli) were aligned along with the DNA nucleotide sequences of flaB from seven of the same isolates (six isolates of $C$. jejuni and one isolate of C. coli). The homoplasy index 


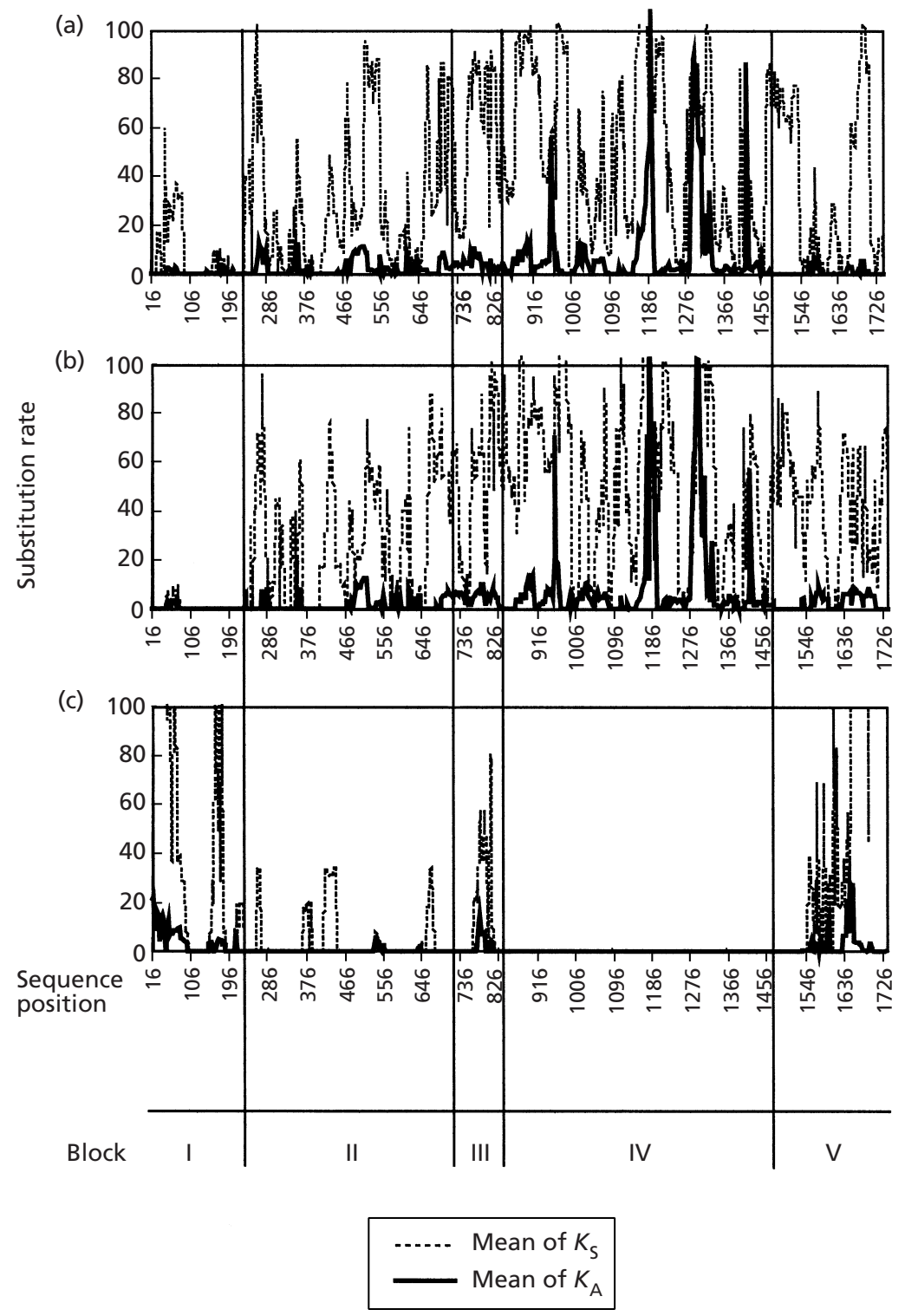

Fig. 2. Site analyses of synonymous $\left(K_{s}\right)$ and non-synonymous $\left(K_{A}\right)$ base substitutions in (a) $f l a A$ for multiple strain sequence alignments, (b) flaB for multiple strain sequence alignments and (c) flaA to fla $B$ within individual strains. The dashed lines represent the density of the synonymous base substitutions and the solid lines represent the density of non-synonymous substitutions. Note that the abscissae indicate the centre of the window site.

was determined for every pair of all the sequences. It was found that the homoplasy indexes for paired $f l a B$ genes were greater than for $f l a A / f l a A$ or $f l a A / f l a B$ pairs, both interstrain and intrastrain, except in pairs of the three flaB sequences that were highly similar (Table 1). D2290, L17 and NCTC 1168 differed by only 2 bp each and are thus expected to have low homoplasy indexes with each other.

Analyses were performed to determine the segments of the sequences that contributed to the homoplasy. The alignment of flaA DNA sequences from 16 strains and the flaB DNA sequences from seven of the same strains was subjected to the Sawyer's runs test (Sawyer, 1989). Using the most conservative method of determining $P$ values, 104 possible gene conversion events were identified with $P<0 \cdot 05$. Sixteen of these were between the $f l a A$ and $f l a B$ of the same strain and the sites of these events are mapped in Fig. 1. The majority of the postulated recombination events were found in the segment of the flagellin genes that we later identify as Block IV (see below). Possible flaA to flaA interstrain conversion events are also shown and reflect those seen by Harrington et al. (1997).

Patterns of synonymous versus non-synonymous base changes may indicate the nature of selective forces on structural genes. Window analyses of synonymous and non-synonymous base substitutions (Li, 1993; Reeves et $a l ., 1994)$ for flaA and flaB were scored separately and are presented in Fig. 2(a,b). The areas of sequence variability between isolates of Campylobacter are very similar for $f l a A$ and $f l a B$. The sites of intrastrain synonymous and non-synonymous substitutions in the $f l a A$ and $f l a B$ sequences for each isolate for which data were available for both genes (seven pairs) were analysed and the results are shown in Fig. 2(c). The sites program (distributed by Jody Hey, Rutgers University) gives a precise location of base substitutions and was used to delimit the blocks that are indicated in Fig. 2. The 
Table 2. Summary of synonymous substitution rate $\left(K_{\mathrm{s}}\right)$, non-synonymous substitution rate $\left(K_{\mathrm{A}}\right)$ and $K_{\mathrm{S}}$ to $K_{\mathrm{A}}$ ratio for blocks of aligned flagellin sequence

Pairwise $K_{\mathrm{S}}$ and $K_{\mathrm{A}}$ were determined by the algorithm developed by Li (1993) and averaged for all the interstrain $f l a A$ comparisons, all the interstrain $f l a B$ comparisons and all the intrastrain $f l a A / f l a B$ comparisons. The $K_{\mathrm{S}}$ and $K_{\mathrm{A}}$ are window-size-dependent, especially for small windows such as Block III. Comparisons should be made across the rows, not down the columns. However, the $K_{\mathrm{S}} / K_{\mathrm{A}}$ ratio should not be affected by window size.

\begin{tabular}{|c|c|c|c|c|c|c|c|c|c|}
\hline \multirow[t]{2}{*}{ Block* } & \multicolumn{3}{|c|}{$f l a A$} & \multicolumn{3}{|c|}{$f l a B$} & \multicolumn{3}{|c|}{$f l a A-f l a B$} \\
\hline & $\boldsymbol{K}_{\mathrm{s}}$ & $K_{\mathrm{A}}$ & $K_{\mathrm{S}} / \boldsymbol{K}_{\mathrm{A}}$ & $\boldsymbol{K}_{\mathrm{s}}$ & $K_{\mathrm{A}}$ & $\boldsymbol{K}_{\mathrm{S}} / \boldsymbol{K}_{\mathrm{A}}$ & $\boldsymbol{K}_{\mathrm{s}}$ & $K_{\mathrm{A}}$ & $\boldsymbol{K}_{\mathrm{S}} / \boldsymbol{K}_{\mathrm{A}}$ \\
\hline All (1-end) & 0.476 & $0 \cdot 106$ & $4 \cdot 49$ & $0 \cdot 454$ & $0 \cdot 100$ & $4 \cdot 54$ & $0 \cdot 159$ & $0 \cdot 047$ & $3 \cdot 47$ \\
\hline I $(1-243)$ & $0 \cdot 249$ & $0 \cdot 032$ & $7 \cdot 78$ & $0 \cdot 149$ & $0 \cdot 012$ & $12 \cdot 42$ & $0 \cdot 608$ & $0 \cdot 151$ & $4 \cdot 03$ \\
\hline II (244-735) & $0 \cdot 423$ & $0 \cdot 071$ & $5 \cdot 96$ & $0 \cdot 383$ & $0 \cdot 065$ & $5 \cdot 89$ & $0 \cdot 040$ & $0 \cdot 010$ & $4 \cdot 24$ \\
\hline III (736-831) & $8 \cdot 104$ & 0.662 & $12 \cdot 24$ & $17 \cdot 357$ & $1 \cdot 266$ & $13 \cdot 71$ & $0 \cdot 351$ & $0 \cdot 109$ & $3 \cdot 21$ \\
\hline IV (832-1515) & 0.650 & $0 \cdot 199$ & $3 \cdot 27$ & $0 \cdot 671$ & $0 \cdot 197$ & $3 \cdot 41$ & $0 \cdot 008$ & $0 \cdot 006$ & $1 \cdot 38$ \\
\hline V (1516-end) & $0 \cdot 372$ & $0 \cdot 025$ & $14 \cdot 88$ & $0 \cdot 461$ & $0 \cdot 013$ & $35 \cdot 46$ & 0.955 & $0 \cdot 148$ & $6 \cdot 43$ \\
\hline
\end{tabular}

*Block equals the segment of the aligned sequence for which data were analysed (base pair positions shown in parentheses).

\begin{tabular}{|c|c|c|c|c|c|c|}
\hline $\begin{array}{l}\text { Consensus } \\
\text { flaA } \\
\text { flaB }\end{array}$ & $\begin{array}{l}\text { MGFRINTN. } 0 \\
\ldots \ldots \ldots \text { X0 } \\
\ldots \ldots \ldots \text { IG }\end{array}$ & $\begin{array}{l}\text { OLNO20NO.3 } \\
\text { A. AKO. ODL } \\
0 \ldots \text { OHA.SVV }\end{array}$ & $\begin{array}{l}\text { N0 . LD.SLO } \\
.020 \ldots X . .0 \\
.0 X E \ldots \text {. . }\end{array}$ & $\begin{array}{c}\text { RLSSGLRINS } \\
\ldots \ldots \ldots \ldots \\
\ldots \ldots \ldots\end{array}$ & $\begin{array}{c}\text { AAD.ASGMAI } \\
\ldots \mathrm{X} \ldots \ldots \\
\ldots \mathrm{D} \ldots\end{array}$ & $\begin{array}{l}150 \\
150 \\
150\end{array}$ \\
\hline $\begin{array}{l}\text { Consensus } \\
\text { flaA } \\
\text { flaB }\end{array}$ & 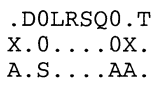 & $\begin{array}{l}\text { LGQAI.NGND } \\
\ldots \text {.s. . } \\
\ldots \text {.N. . }\end{array}$ & $\begin{array}{l}\text { A3GILQTADK } \\
.3 \ldots \ldots \\
.1 \ldots \ldots\end{array}$ & $\begin{array}{c}\text { AMDEQLKILD } \\
\ldots \ldots \cdots \\
\ldots \ldots\end{array}$ & $\begin{array}{l}\text { TIKTKATQAA } \\
\cdots \cdots \cdots \\
\ldots \ldots \\
\end{array}$ & $\begin{array}{l}300 \\
300 \\
300\end{array}$ \\
\hline $\begin{array}{l}\text { Consensus } \\
\text { flaA } \\
\text { flaB }\end{array}$ & $\begin{array}{l}\text { 1DGQSLKTRT } \\
1 \ldots \ldots \ldots \\
Q \ldots \ldots \ldots\end{array}$ & $\begin{array}{l}\text { MLQA1IN2LM } \\
\ldots \ldots 1 \ldots 2 \ldots \\
\ldots \text { D. R. }\end{array}$ & $\begin{array}{c}\text { EELDNIANTT } \\
\cdots \cdots \cdots \\
\cdots \cdots \cdots\end{array}$ & $\begin{array}{c}\text { SF.GKQLLSG } \\
\ldots \mathrm{X} . \ldots \ldots \\
\ldots \mathrm{N} . \ldots \ldots\end{array}$ & $\begin{array}{l}\text {.F.NQEFQIG } \\
\mathrm{X} . \mathrm{X} . \ldots \ldots \\
\mathrm{X} . \mathrm{X} \ldots \ldots\end{array}$ & $\begin{array}{l}450 \\
450 \\
450\end{array}$ \\
\hline $\begin{array}{l}\text { Consensus } \\
\text { flaA } \\
\text { flaB }\end{array}$ & $\begin{array}{l}\text { 0SSNQT3K00 } \\
0 \ldots \ldots 3.00 \\
0 \ldots \ldots 3 . \text { A0 }\end{array}$ & $\begin{array}{c}\text { IGATQSSKIG } \\
\cdots \ldots \ldots \\
\ldots \ldots \ldots\end{array}$ & $\begin{array}{l}3 \mathrm{TR} .1 \ldots \ldots \\
3 \ldots \mathrm{X} 1 \mathrm{XXXXX} \\
3 \ldots \mathrm{FETGXXX}\end{array}$ & $\begin{array}{l}\ldots G \cdot V . T 3 \\
\text { XXX.X.XX.3 } \\
\text { XX0.X.XX.3 }\end{array}$ & $\begin{array}{l}\text { KNYNGI1DF. } \\
\ldots \ldots 1 \ldots \mathrm{X} \\
\ldots \ldots 1 \ldots \mathrm{X}\end{array}$ & $\begin{array}{l}600 \\
600 \\
600\end{array}$ \\
\hline $\begin{array}{l}\text { Consensus } \\
\text { flaA } \\
\text { flaB }\end{array}$ & $\begin{array}{l}\text { F1.VVISTSV } \\
.1 \mathrm{x} \ldots \ldots \\
.1 \mathrm{x} \ldots \ldots\end{array}$ & $\begin{array}{l}\text { GTGLGALA1E } \\
\ldots \ldots \ldots 1 . \\
\ldots \ldots \ldots \text { E. }\end{array}$ & $\begin{array}{l}\text { IN2.AD.TG3 } \\
\ldots 2 \mathrm{X} . \mathrm{K} .3 \\
\ldots 2 \mathrm{x} . \mathrm{x} .3\end{array}$ & $\begin{array}{l}\text { RAT4.3...G } \\
\ldots 4 \text { X3XXX. } \\
\ldots 4 \text { XVX0X. }\end{array}$ & $\begin{array}{l}\text {.A32.G.TS } \\
\mathrm{XX} .32 \mathrm{X} . \mathrm{X} . \\
\mathrm{XX} .32 \mathrm{X} . \mathrm{X} .\end{array}$ & $\begin{array}{l}750 \\
750 \\
750\end{array}$ \\
\hline $\begin{array}{l}\text { Consensus } \\
\text { flaA } \\
\text { flaB }\end{array}$ & $\begin{array}{l}1 . \mathrm{F} 0 \text { INGV.I } \\
1 \mathrm{X} .0 \ldots \mathrm{X} . \\
1 \mathrm{X} . \mathrm{A} \ldots \mathrm{X} .\end{array}$ & $\begin{array}{l}\text { G.3.Y.DGD. } \\
. X 3 X \cdot X \ldots X \\
. Q 3 X . X \ldots X\end{array}$ & $\begin{array}{l}\text { NG.L30AINO } \\
\ldots X .30 \ldots 0 \\
\ldots X .30 \ldots 0\end{array}$ & $\begin{array}{c}\text { VKDTTGV1AS } \\
\ldots \ldots 1 . \\
\ldots \ldots 1\end{array}$ & $\begin{array}{l}\text { D.NG.L3LT } \\
\text { X.X.X. } 3 \cdots \\
3 . X . X .3 \ldots\end{array}$ & $\begin{array}{l}900 \\
900 \\
900\end{array}$ \\
\hline $\begin{array}{l}\text { Consensus } \\
\text { flaA } \\
\text { flaB }\end{array}$ & $\begin{array}{l}\text { S.1GRGIKI. } \\
. \mathrm{X} 1 \ldots \ldots \mathrm{X} \\
. \mathrm{X} 1 \ldots \ldots \mathrm{X}\end{array}$ & $\begin{array}{l}\text { G.IG.GO.I. } \\
. X . X .0 X . X \\
\text { X.X.OX.X }\end{array}$ & $\begin{array}{l}\ldots \ldots \text { ENYGRL } \\
\mathrm{XXXX} \ldots \ldots \\
\mathrm{X} 1 \mathrm{XX} \ldots \ldots\end{array}$ & $\begin{array}{c}\text { SLV.KNDG21 } \\
\ldots \mathrm{X} \ldots \ldots 2 \mathrm{D} \\
\ldots \ldots . \ldots 21\end{array}$ & 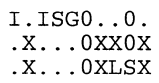 & $\begin{array}{l}1050 \\
1050 \\
1047\end{array}$ \\
\hline $\begin{array}{l}\text { Consensus } \\
\text { flaA } \\
\text { flaB }\end{array}$ & $\begin{array}{l}\text { G.G...S } \\
X . X . X X X X X . \\
\text { X.X.001XI. }\end{array}$ & $\begin{array}{l}\text { Q.SVSLRESK } \\
. \mathrm{X} \ldots \ldots \ldots \\
. \mathrm{X} \ldots \ldots\end{array}$ & $\begin{array}{l}\text { G..A..ADA } \\
. \mathrm{XXX} \cdot \mathrm{XX} \ldots \\
. \mathrm{XXX} \cdot \mathrm{XX} \ldots\end{array}$ & $\begin{array}{l}\text { MGF.0...G. } \\
\ldots \text { X0XXX.X } \\
\ldots \text { X0XXX.X }\end{array}$ & $\underset{\mathrm{xxxxx}}{\ldots \ldots \ldots \ldots x x x x x}$ & $\begin{array}{l}1200 \\
1200 \\
1197\end{array}$ \\
\hline $\begin{array}{l}\text { Consensus } \\
\text { flaA } \\
\text { flaB }\end{array}$ & $\begin{array}{l}\text { S030.4MS .. } \\
.030 \times 4 \ldots \mathrm{XX} \\
. \mathrm{S} 30 \mathrm{X} 4 \ldots \mathrm{XX}\end{array}$ & $\begin{array}{l}\text {.SGFS.GSG } \\
X \ldots \ldots \times 4 \\
G \ldots \ldots X \ldots X\end{array}$ & $\begin{array}{l}\text { SVGSGK..S. } \\
\ldots \ldots \mathrm{XX} . \mathrm{X} \\
\ldots \ldots \mathrm{XX} . \mathrm{X}\end{array}$ & 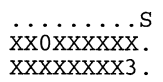 & $\begin{array}{l}0.0 .3 S \ldots Y \\
0 X 0 X 3 . X X . X \\
0 A 0 X 3 . X X . X\end{array}$ & $\begin{array}{l}1350 \\
1350 \\
1347\end{array}$ \\
\hline $\begin{array}{l}\text { Consensus } \\
\text { flaA } \\
\text { flab }\end{array}$ & $\begin{array}{l}\text { 3S.GSGFS0. } \\
3 . \mathrm{X} . \ldots 0 \mathrm{X} \\
\mathrm{V} . \mathrm{X} \ldots . .0 \mathrm{G}\end{array}$ & $\begin{array}{l}\text { S..SQF0.32 } \\
. \mathrm{XX} . .0 \times 32 \\
. \mathrm{XX} . . \mathrm{A} 032\end{array}$ & $\underset{\mathrm{x} 00 \mathrm{xxxxxxx}}{0 \ldots \ldots \ldots}$ & $\begin{array}{c}\text { DETAGVTTLK } \\
\cdots \cdots \cdots \\
\cdots \cdots \cdots\end{array}$ & $\begin{array}{l}\text { GAMA.MDIAE } \\
\ldots . \mathrm{X} . \ldots \\
\ldots . \mathrm{V} . \ldots\end{array}$ & $\begin{array}{l}1500 \\
1500 \\
1497\end{array}$ \\
\hline $\begin{array}{l}\text { Consensus } \\
\text { flaA } \\
\text { flaB }\end{array}$ & $\begin{array}{l}\text { T. .TN.DQIR } \\
. A X \ldots L \ldots \\
. \mathrm{XX} . \mathrm{X} \ldots\end{array}$ & $\begin{array}{l}\text { ADIG03QNQ3 } \\
\ldots . \mathrm{S} 3 \ldots 3 \\
\ldots . \mathrm{OV} \ldots \mathrm{L}\end{array}$ & $\begin{array}{l}\ldots \text { TINNITVT } \\
\text { Ts. } \ldots \ldots \ldots \\
\text { QV. } \ldots \ldots \ldots\end{array}$ & $\begin{array}{l}\text { QVNVKOAES. } \\
\ldots \ldots 0 \ldots \text { Q } \\
\ldots \ldots \text { A. }\end{array}$ & $\begin{array}{l}\text { IRDVDF } 00 \mathrm{ES} \\
\ldots \ldots 00 \ldots \\
\ldots \ldots \text { A0 }\end{array}$ & $\begin{array}{l}1650 \\
1650 \\
1647\end{array}$ \\
\hline $\begin{array}{l}\text { Consensus } \\
\text { flaA } \\
\text { flaB }\end{array}$ & $\begin{array}{l}\text { ANASK.NILA } \\
\ldots \mathbf{Y} \ldots \mathbf{A} \ldots \\
\ldots \mathbf{F} . \mathbf{Y} \ldots\end{array}$ & $\begin{array}{l}\text { QSGS.OMOQ. } \\
\ldots \text {.XOA.A } \\
\ldots \text {.YA.S.X }\end{array}$ & $\begin{array}{l}\text { NO . QNVL2L } \\
\text {. SXX . . R. } \\
\text {.AVQ . . K. }\end{array}$ & $\begin{array}{l}L Q \\
\cdots \\
\cdots\end{array}$ & & $\begin{array}{l}1746 \\
1746 \\
1743\end{array}$ \\
\hline
\end{tabular}

\begin{abstract}
Fig. 3. Comparison of the consensus sequence of translated amino acid sequence of flaA with the consensus sequence of translated flaB. The sequence indicated 'Consensus' shows the residues in common between the flaA and flaB consensus. Residues indicated in bold are sites that are highly conserved in both sequences but differ between flaA and flaB. Numbering on the right is the nucleotide position of the corresponding DNA sequence. Numbers substituted for the amino acid designation are the pam250S scores: 0 for Ala, Gly, Pro, Ser, Thr; 1 for Asp, Glu, Asn, Gln, Asx, Glx; 2 for His, Lys, Arg; 3 for Ile, Leu, Met, Val; 4 for Phe, Trp, Tyr; or 5 for Cys.
\end{abstract}

greatest variability of flaA compared to flaB of the same isolate occurs in three regions, one close to the $5^{\prime}$ terminus of the DNA sequence, a short segment at the centre of the sequence, and another region at the $3^{\prime}$ end of the sequence. Five blocks with differing patterns of base substitutions in the flagellin gene sequence were identified as follows: Block I, base positions 1-243; Block II, base positions 244-735; Block III, base positions 736-831; Block IV, base positions 832-1515;
Block V, base positions 1516 to end. The mean synonymous substitution rate $\left(K_{\mathrm{S}}\right)$, non-synonymous substitution rate $\left(K_{\mathrm{A}}\right)$ and the $K_{\mathrm{S}}$ to $K_{\mathrm{A}}$ ratio were calculated for pairwise comparisons (120 interstrain $f l a A$ to flaA comparisons, 21 flaB to flaB interstrain comparisons and seven intrastrain flaA to $f l a B$ comparisons) for each block and are presented in Table 2. Because $K_{\mathrm{S}}$ and $K_{\mathrm{A}}$ calculations become sensitive to window size as the window size gets small (compare 
data in Table 2 with scales for Fig. 2, which are for windows of $30 \mathrm{bp}$ ), the numbers should be compared across the table and not up and down the columns.

Consensus sequences for the predicted amino acid sequences of $f l a A$ and $f l a B$ were constructed. Any residue position that did not give a $100 \%$ consensus was scored using the pam250S score for conserved amino acid side chain structure (Dayhoff et al., 1978). Residues that did not match a conservation group were scored as not having a consensus. The scored consensus amino acid sequences for FlaA and FlaB were then aligned (Fig. 3). Only 12 strictly conserved differences were observed, i.e. a specific amino acid was found in all the FlaAs and a different specific amino acid was found in all the FlaBs. Six of these 12 differences were pairs of amino acids that were in the same pam250S groups. Four of the 12 differences were within 76 amino acid residues of the amino-terminal end of the protein. The remaining differences were within 62 residues of the carboxyterminus.

\section{DISCUSSION}

Homoplasy is considered a departure from random mutations, and this departure may be due to parallel or convergent evolution or evolutionary reversals (Swofford et al., 1996; Stewart, 1993). The homoplasy index is derived from phylogenetic trees to indicate the frequency of matching character states that are not found in putative ancestors. Phylogenetic trees are not usually considered valid lineage reconstructions when paralogous genes are included in the analysis (Swofford et al., 1996). Nevertheless, our analyses showed that the homoplasy index was a useful tool in this study. The $f l a A$ and $f l a B$ sequences from the same isolates clustered together in dendrograms (not shown). This is an indication of concerted evolution ( $\mathrm{Li}, 1997)$. However, the homoplasy index also revealed associations of $\mathrm{flaB}$ between strains. Pairwise comparisons of flaA and flaB sequences within isolates then revealed regions (at least Block IV, defined above) of apparent concerted evolution. The Sawyer's runs test supported this hypothesis by showing that Block IV appeared as a possible recombinant fragment between fla $A$ and $f l a B$ in all the strains for which both sequences were available.

The extremely low number of intrastrain $f l a A$ to $f l a B$ synonymous differences in the region of DNA sequence bases 832 and 1515 between the flaA and $f l a B$ of individual strains implies editing or gene conversion with selection at the DNA level (Endo et al., 1996). The higher level of intrastrain flaA to flaB synonymous differences between bases 244 and 735 suggests selection for conservation at the protein or functional level without gene conversion (Endo et al., 1996). However, the observed rates for this region could be within the range that might occur in parts of the Campylobacter genome that do not have selection for diversity. No studies of multiple-sequence analyses of Campylobacter housekeeping genes have yet been published that will allow this comparison to be made. From the $K_{\mathrm{S}} / K_{\mathrm{A}}$ ratios in Table 2 it appears that there is greatest functional constraint on sequence in Block $\mathrm{V}$ and the lowest functional constraint in Block II and Block IV. Block IV is the segment that has almost no differences between the $f l a A$ and $f l a B$ within individual strains. However, even with the degree of variability seen, one constraint that was maintained was the $\mathrm{G}+\mathrm{C}$ content. The $\mathrm{G}+\mathrm{C}$ content of the fla sequences from C. jejuni strains used in this study was from 35.0 to $37 \cdot 2 \mathrm{~mol} \%$ (mean $36.4 \mathrm{~mol} \%$ ).

Recombinant shuffling of the variable regions of flaA and $f l a B$ within a strain would not itself generate interstrain variability with intrastrain conservation. In fact, the rate of concerted evolution is expected to be higher when there are structural constraints (Li, 1997). Recombination errors that may increase variability should occur at the recombination junctions. If this was occurring with $f l a$, then it is expected that the interstrain variability would be concentrated at the ends of the region that is intrastrain conserved. Alignment gaps were seen that are consistent with illegitimate recombinant events (Jakupciak \& Wells, 1999). However, we have found that interstrain variability is greatest within the segment that is conserved intrastrain, perhaps indicating that the generation of diversity in that region is not recombination-dependent.

Campylobacter is competent for transformation (Wang \& Taylor, 1990) and does not appear to be clonal (Aeschbacher \& Piffaretti, 1989). Therefore, it is possible that generation of variability may involve recombinant shuffling of segments of fla between strains. Harrington et al. (1997) presented convincing evidence of interstrain recombination within the fla $A$ gene. There would then need to be a simultaneous or sequential insertion of the new sequence into the analogous segment of the other copy of fla within the strain. Intrastrain recombinant events between the fla $A$ and $f l a B$ that cause a loss of the interstrain-conserved regions, i.e. the regions that distinguish fla $A$ from $f l a B$ within a strain, would have to be treated as an error and lost (purged). Unequal crossing-over appears to be a prevalent mechanism of concerted evolution in eukaryotes $(\mathrm{Li}, 1997)$. It is unlikely that unequal crossing-over occurs with Campylobacter fla because the mechanism usually involves changes in the copy number. Precision splicing of regions of the genes between $f l a A$ and $f l a B$ could occur, but putative insertion sequences or chi sequences (West, 1992) that would facilitate such a mechanism were not conserved within the fla sequences examined in this study. Also, recombination between the two copies of the flagellin would have had to invoke some copy-choice mechanism that favoured the low $\mathrm{G}+\mathrm{C}$ content that is characteristic of the Campylobacter genome. That is to say, if a mutation occurred in one copy of the flagellin that substituted a new G or C, the amelioration process of the two copies would need to favour the A or T copy. Since transitional nucleotide substitutions are biochemically more favoured than transversions ( $\mathrm{Li}, 1997)$, failure to do so would drive towards a $50 \% \mathrm{G}+\mathrm{C}$ content ratio. Alternatively, there 
may be non-replicative mechanisms for conversion of one fla sequence based on the other fla sequence such as a mismatch repair mechanism. If there was no preference for which version of the gene was maintained, either mechanism, recombination or mismatch repair, might increase the rate of generation of diversity of the genes within a population of the organism. Some progeny of a cell with a mutation in the intrastrain conserved segment would end up with the version that was originally fla $A$ and others would end up with the $f l a B$ version. Gene conversion using mismatch repair of the two genes might also allow for simpler mechanisms that could favour adenosine or thymidine. The mean interstrain differences for flaA were slightly higher (about $162 \mathrm{bp}$ difference) than the interstrain $f l a B$ differences (about $159 \mathrm{bp}$ ). If changes usually occurred first in $f l a A$ and are later transferred to $f l a B$, a higher number of interstrain differences would be expected in flaA. That conclusion can not be drawn from the data because the differences are not great enough.

Examination of the amino acid sequence differences between the consensus sequences of FlaA and FlaB may explain why two copies of flagellin are maintained. In the amino acid sequence from residue 290 to residue 480 , only 71 of the residues were conserved in the aligned FlaA or FlaB amino acid sequences that were analysed. This is an indication that the flagellin protein has little functional dependence on primary amino acid sequence in that region. This is the region, however, that is maintained between the $f l a A$ and $f l a B$ within an individual strain and, based on homology with the Salmonella flagellin, is expected to be the bearer of surface-exposed epitopes (Yamashita et al., 1995). Furthermore, the high homoplasy indexes for flaBs imply a stronger selective pressure on $f l a B$ than on flaA. Six of the 12 amino acids that are strictly conserved in each copy of FlaA but differ between FlaA to FlaB represent differences in amino acids that can be O-linked (serine or threonine). Five of these modifiable amino acids are in the carboxy-terminal region. Doig et al. (1996) have demonstrated that Campylobacter flagellins are glycosylated. It would seem that the different copies of the flagellin gene offer the greatest opportunity for antigenic phase variation by changing the glycosylation sites.

Copy-to-copy conversion can increase the mutation rate of the gene by bringing together the mutations from two sites. This would contribute to clonal differences of the organism but at the expense of the ability to have greater phase variation. The advantage of maintaining two copies of the flagellin gene remain unclear, but it would be efficient if they contributed both to function or phase variation and to increasing the genetic pool for producing greater clone-to-clone variation. Presumably, host immune responses would exert selection for diversity that would drive the expansion of new alleles of flagellin. The two independent promoters for flaA and flaB (Guerry et al., 1990; Nuijten et al., 1990) would allow a mechanism for phase variation, but the host factors that might activate either promoter are not known.
The pattern of concerted evolution noted in this study is unusual for prokaryotes. Most Salmonella maintain two copies of flagellin genes, $f l i C$ and $f l j B$. Okazaki et al. (1993) demonstrated exchange of genetic information between the phase variant flagellin genes $f l i C$ and $f l j B$ of $S$. typhimurium after selection against expression of parental fliC epitopes. Neisseria gonorrhoeae uses gene conversion to exchange sequence between the expressed pilin gene and non-expressed pseudogenes (Zhang et al., 1992). We are not aware of any study to analyse the Salmonella flagellin or Neisseria pilin gene families for concerted evolution.

Concerted evolution is defined as less than expected divergence of copies of genes within an individual than the divergence of the gene from other species $(\mathrm{Li}, 1997)$. We have demonstrated that concerted evolution of segments of Campylobacter fla appears to occur at a rate that at least equals the rate of clonal divergence. There is no information currently available on the divergence rate of other genes in Campylobacter. Flagellin clearly has a greater diversity than expected for most of the genome. Thus it can be concluded that the intrastrainconserved region (Block IV) in Campylobacter fla demonstrates concerted evolution relative to the remainder of the flagellin gene, but we do not know if this is true relative to the entire genome. The concerted evolution occurring in Block IV must happen by gene conversion events that occur almost as fast as the mutation rate since the Block IV sequences of fla $\mathrm{A}$ and $f l a B$ are almost identical within a strain.

\section{ACKNOWLEDGEMENTS}

The material presented in this paper is the product of the official duties of the authors as employees of the USDA Agricultural Research Service. No endorsement is expressed or implied by the Agency for any products that are mentioned. The authors gratefully acknowledge the clarifying comments made by an anonymous reviewer.

\section{REFERENCES}

Abdulkarim, F. \& Hughes, D. (1996). Homologous recombination between the tuf genes of Salmonella typhimurium. J Mol Biol 260, 506-522.

Aeschbacher, M. \& Piffaretti, J. C. (1989). Population genetics of human and animal enteric Campylobacter isolates. Infect Immun 57, 1432-1437.

Alm, R. A., Guerry, P. \& Trust, T. J. (1993). Significance of duplicated flagellin genes in Campylobacter. J Mol Biol 230, 359-363.

Dayhoff, M., Schwartz, R. M. \& Orcutt, B. C. (1978). Atlas of Protein Sequence and Structure, vol. 5, suppl. 3, p. 345. Silver Spring, MD : National Biomedical Research Foundation.

Doig, P., Kinsella, N., Guerry, P. \& Trust, T. J. (1996). Characterization of a post-translational modification of Campylobacter flagellin: identification of a sero-specific glycosyl moiety. Mol Microbiol 19, 379-387.

Endo, T., Ikeo, K. \& Gojobori, T. (1996). Large-scale search for genes on which positive selection may operate. Mol Biol Evol 13, 685-690. 
Guerry, P., Logan, S. M., Thornton, S. \& Trust, T. J. (1990). Genomic organization and expression of Campylobacter flagellin genes. J Bacteriol 172, 1853-1860.

Harrington, C. S., Thomson-Carter, F. M. \& Carter, P. E. (1997). Evidence for recombination in the flagellin locus of Campylobacter jejuni: implications for the flagellin gene typing scheme. $J$ Clin Microbiol 35, 2386-2392.

Jakupciak, J.P. \& Wells, R. D. (1999). Genetic instabilities in (CTG.CAG) repeats occur by recombination. J Biol Chem 274 23468-23479.

Jensen, R. A. \& Gu, W. (1996). Evolutionary recruitment of biochemically specialized subdivisions of Family I within the protein superfamily of aminotransferases. J Bacteriol 178, 21612171.

Khawaja, R., Neote, K., Bingham, H. L., Penner, J. L. \& Chan, V. L. (1992). Cloning and sequence analysis of the flagellin gene of Campylobacter jejuni TGH9011. Curr Microbiol 24, 213-221.

Kowalchuk, G. A., Gregg-Jolly, L. A. \& Ornston, L. N. (1995). Nucleotide sequences transferred by gene conversion in the bacterium Acinetobacter calcoaceticus. Gene 153, 111-115.

Li, W.-H. (1993). Unbiased estimation of the rates of synonymous and nonsynonymous substitutions. J Mol Evol 36, 96-99.

Li, W.-H. (1997). Molecular Evolution. Sunderland, MA: Sinauer Associates.

Logan, S. M., Trust, T. J. \& Guerry, P. (1989). Evidence for posttranslational modification and gene duplication of Campylobacter flagellin. J Bacteriol 169, 3031-3038.

Madoff, L. C., Michel, J. L., Gong, E. W., Kling, D. E. \& Kasper, D. L. (1996). Group B streptococci escape host immunity by deletion of tandem repeat elements of the alpha C protein. Proc Natl Acad Sci USA 93, 4131-4136.

Mattatall, N. R., Daines, D. A., Liu, S.-L. \& Sanderson, K. E. (1996) Salmonella typhi contains identical intervening sequences in all seven $\mathrm{rrl}$ genes. J Bacteriol 178, 5323-5326.

Meinersmann, R. J., Helsel, L. O., Fields, P. I. \& Hiett, K. L. (1997). Discrimination of Campylobacter jejuni by fla gene sequencing. J Clin Microbiol 35, 2810-2814.

Nachamkin, I., Yang, X. H. \& Stern, N. J. (1993). Role of Campylobacter jejuni flagella as colonization factors for three day old chicks: analysis with flagellar mutants. Appl Environ Microbiol 59, 1269-1273.

Nuijten, P. J., van Asten, F. J., Gaastra, W. \& van der Zeijst, B. A. (1990). Structural and functional analysis of two Campylobacter jejuni flagellin genes. J Biol Chem 265, 17798-17804.
Okazaki, N., Matsuo, S., Saito, K., Tominaga, A. \& Enomoto, M. (1993). Conversion of the Salmonella phase 1 flagellin gene $f l i C$ to the phase $2 f l j B$ on the Escherichia coli $\mathrm{K}-12$ chromosome. $J$ Bacteriol 175, 758-766.

Reeves, P. R., Farnell, L. \& Lan, R. (1994). multicomP : a program for preparing sequence data for phylogenetic analysis. Comput Appl Biosci 10, 281-284.

Sawyer, S. A. (1989). Statistical tests for detecting gene conversion. Mol Biol Evol 6, 526-538.

Stewart, C. B. (1993). The powers and pitfalls of parsimony. Nature 361, 603-607.

Swofford, D. L. (1999). PAUP*. Phylogenetic analysis using parsimony ("and other methods), version 4. Sunderland, MA, Sinauer Associates.

Swofford, D. L., Olsen, G. J., Waddell, P. J. \& Hillis, D. M. (1996). Phylogenetic inference. In Molecular Systematics, 2nd edn, pp. 407-514. Edited by D. M. Hillis, C. Moritz \& B. K. Mable. Sunderland, MA: Sinauer Associates.

Thompson, J. D., Higgins, D. G. \& Gibson, T. J. (1994). CLUSTAL W : improving the sensitivity of progressive multiple sequence alignment through sequence weighting, position specific gap penalties and weight matrix choice. Nucleic Acids Res 22, 4673-4680.

Wang, Y. \& Taylor, D. E. (1990). Natural transformation in Campylobacter species. J Bacteriol 172, 949-955.

Wassenaar, T. M., Bleumink-Pluym, N. M. C. \& van der Zeijst, B. A. M. (1991). Inactivation of Campylobacter jejuni flagellin genes by homologous recombination demonstrates that fla $A$ but not $f l a B$ is required for invasion. EMBO J 10, 2055-2061.

Wassenaar, T. M., van der Zeijst, B. A., Ayling, R. \& Newell, D. G. (1993). Colonization of chicks by motility mutants of Campylobacter jejuni demonstrates the importance of flagellin A expression. J Gen Microbiol 139, 1171-1175.

West, S. C. (1992). Enzymes and molecular mechanisms of genetic recombination. Annu Rev Biochem 61, 603-640.

Yamashita, I., Vonderviszt, F., Mimori, Y., Suzuki, H., Oosawa, K. \& Namba, K. (1995). Radial mass analysis of the flagellar filament of Salmonella: implications for the subunit folding. J Mol Biol 253, 547-558.

Zhang, Q. Y., DeRyckere, D., Lauer, P. \& Koomey, M. (1992). Gene conversion in Neisseria gonorrhoeae: evidence for its role in pilus antigenic variation. Proc Natl Acad Sci USA 89, 5366-5370.

Received 18 February 2000; revised 19 May 2000; accepted 26 May 2000. 